Draft Version SEPTEMBER 15, 2018

Preprint typeset using LTEX style emulateapj v. 5/2/11

\title{
CARBON-ENHANCED METAL-POOR STARS: RELICS FROM THE DARK AGES
}

\author{
Ryan J. COOKE ${ }^{1,2}$ AND Piero Madau ${ }^{1}$ \\ (Dated: September 15, 2018) \\ Draft version September 15, 2018
}

\begin{abstract}
We use detailed nucleosynthesis calculations and a realistic prescription for the environment of the first stars to explore the first episodes of chemical enrichment that occurred during the dark ages. Based on these calculations, we propose a novel explanation for the increased prevalence of carbon-enhanced metal-poor (CEMP) stars with decreasing Fe abundance: The observed chemistry for the most metal-poor Galactic halo stars is the result of an intimate link between the explosions of the first stars and their host minihalo's ability to retain its gas. Specifically, high-energy supernovae produce a near solar ratio of $\mathrm{C} / \mathrm{Fe}$, but are effective in evacuating the gas from their host minihalo, thereby suppressing the formation of a second generation of stars. On the other hand, minihalos that host low-energy supernovae are able to retain their gas and form a second stellar generation but, as a result, the second stars are born with a supersolar ratio of $\mathrm{C} / \mathrm{Fe}$. Our models are able to accurately reproduce the observed distributions of $[\mathrm{C} / \mathrm{Fe}]$ and $[\mathrm{Fe} / \mathrm{H}]$, as well as the fraction of CEMP stars relative to non-CEMP stars as a function of $[\mathrm{Fe} / \mathrm{H}]$ without any free parameters. We propose that the present lack of chemical evidence for very massive stars $\left(\gtrsim 140 \mathrm{M}_{\odot}\right)$ that ended their lives as a highly energetic pairinstability supernova, does not imply that such stars were rare or did not exist; the chemical products of these very massive first stars may have been evacuated from their host minihalo, and were never incorporated into subsequent generations of stars. Finally, our models suggest that the most Fe-poor stars currently known may have seen the enrichment from a small multiple of metal-free stars, and need not have been exclusively enriched by a solitary first star. These calculations also add further support to the possibility that some of the surviving dwarf satellite galaxies of the Milky Way are the relics of the first galaxies.

Subject headings: astrochemistry — cosmology: theory — stars: abundances — stars: chemically peculiar — stars: Population III
\end{abstract}

\section{INTRODUCTION}

The first episodes of star formation arguably mark one of the most important chemical transformations of our Universe, a process where the initially pristine $\mathrm{H}$ and $\mathrm{He}$ nuclei were converted into heavy elements for the first time. Although our current understanding of these first stars is far from complete, the qualitative picture is wellestablished (see e.g. Bromm (2013) for a recent review); the first stars formed in $\sim 10^{6} \mathrm{M}_{\odot}$ dark matter minihalos at redshifts $15 \lesssim z \lesssim 30$ (Haiman, Thoul, \& Loeb 1996; Tegmark et al. 1997), and were predominantly a massive population of stars owing to a lack of efficient cooling routes in the primordial gas. This picture continues to receive strong support from cosmological hydrodynamic simulations (Abel, Bryan, \& Norman 2002; Bromm, Coppi, \& Larson 2002, and other references cited herein). Despite the great success of these foundations, some of the most fundamental properties of the first stars have yet to be pinned down, including the distribution of their stellar mass (Greif et al. 2011; Stacy \& Bromm 2013; Hirano et al. 2014), the mixing properties and energy released during their putative supernova $(\mathrm{SN})$ explosion (Tominaga, Umeda, \& Nomoto 2007; Heger \& Woosley 2010; Limongi \& Chieffi 2012), their binary fraction (Stacy \& Bromm 2013), the distribution of their host minihalo masses (Yoshida et al. 2003; O'Shea \& Norman 2007; Hirano et al. 2014), and the finer details of their chemical enrichment (Madau, Ferrara, \& Rees 2001; $\quad$ Bromm, Yoshida, \& Hernquist 2003;

\footnotetext{
${ }^{1}$ Department of Astronomy and Astrophysics, UCO/Lick Observatory, University of California, Santa Cruz, CA 95064, USA

${ }^{2}$ Morrison/Hubble Fellow; email: rcooke@ucolick.org
}

Scannapieco, Schneider, \& Ferrara 2003; Tumlinson 2006; Wise \& Abel 2008; Komiva et al. 2010; Maio et al. 2011; Ritter et al. 2012).

If metal-free stars (or gas of primordial composition) still exist in the present day Universe, they have not yet been found. At present, the only primordial environments that have been uncovered are at high redshift (Fumagalli. O’Meara, \& Prochaska 2011; Simcoe et al. 2012); the discovery of such systems is crucial for providing a direct physical insight into the formation environments of the first stars. Otherwise, our complete understanding of the first stars relies on simulated universes and other indirect probes, such as the chemical yields from metal-free stars. Fortunately, there now exist a small handful of gas-rich systems at high redshift with metallicities $\lesssim 1 / 1000$ of solar that might be solely enriched by the earliest stellar populations (Pettini et al.|2008; Ellison et al. 2010; Cooke et al. 2011a.b; Cooke, Pettini, \& Murphy 2012). These recent advances at high redshift offer a new and highly complementary approach for studying the nucleosynthesis from the first stars, which has traditionally been pursued by studying the most metal-poor, second generation stars in the local Universe (Cavrel et al. 2004; Lai et al. 2008; Roederer et al. 2014).

Perhaps one of the most striking results that has surfaced from studies of metal-poor Galactic halo stars is the great diversity in their chemical composition. Specifically, around one-quarter of all stars with $\left[\mathrm{Fe} / \mathrm{H}^{3}\right]<-2.0$ exhibit a stark underabundance of heavy elements (e.g. Fe) relative to lighter

3 Throughout this paper, we adopt the standard notation $[\mathrm{X} / \mathrm{Y}] \equiv$ $\log N(\mathrm{X}) / N(\mathrm{Y})-\log (\mathrm{X} / \mathrm{Y})_{\odot}$, where $N(\mathrm{X}) / N(\mathrm{Y})$ is the number abundance ratio of element $\mathrm{X}$ relative to element $\mathrm{Y}$, and the $\odot$ symbol refers to the solar value, taken from Asplund et al. (2009). 
metals (e.g. C) (Beers \& Christlieb 2005; Lucatello et al. 2006; Aoki et al. 2007; Norris et al. 2013; Yong et al. 2013), which becomes even more pronounced at lower $[\mathrm{Fe} / \mathrm{H}]$ abundance. Such stars are collectively known as Carbon-Enhanced Metal-Poor (CEMP 4 stars, and are somewhat arbitrarily defined to have $[\mathrm{C} / \mathrm{Fe}]>+0.7$ (see e.g. Aoki et al. 2007).

Large samples of CEMP stars have shown that there appears to be four distinct sub-classes, which are usually defined on the basis of their neutron-capture elements (see Beers \& Christlieb 2005 for further details): (1) and (2) CEMP- $s$ and CEMP- $r$ stars, which are enhanced in the $s$ process and $r$-process relative to the solar composition, respectively; (3) CEMP-rs stars, which exhibit enhancements in both the $r$ - and $s$ - neutron-capture processes; and (4) CEMPno stars, with a normal abundance pattern for their neutroncapture elements.

Several mechanisms have been proposed to explain the origin of these distinct classes of CEMP stars. For example, the CEMP- $s$ stars are known to be members of a binary system where an asymptotic giant branch (AGB) star transferred carbon-rich (and $s$-process-rich) material onto a low-mass long-lived companion star that we observe today to be anomalously rich in C (e.g. McClure 1985). Indeed, long-term monitoring of the radial velocities of such stars seems to confirm this picture (Lucatello et al. 2005; Cohen et al. 2006; Starkenburg et al. 2014). The origin of the CEMP-no stars, however, is still a matter of debate. While some CEMP-no stars could be an extension of the CEMP- $s$ class to lower metallicity and lower $s$-process enhancement (Campbell \& Lattanzio 2008; Masseron et al. 2010; Karakas \& Lattanzio 2014), there are several lines of evidence that support an alternative origin for the carbon enhancement in some CEMP-no stars. First, the most Fe-poor CEMP-no stars exhibit the largest $\mathrm{C} / \mathrm{Fe}$ ratios (Christlieb et al. 2002; Frebel et al. 2005; Norris et al. 2007; Keller et al. 2014) and appear to be more common at lower metallicities (Beers \& Christlieb 2005; Aoki et al. 2007; Carollo et al. 2012; Yong et al. 2013; Carollo et al. 2014). In addition, the overabundance of each element relative to $\mathrm{Fe}$ decreases with increasing atomic number. This evidence suggests in fact that iron is strongly depressed in these stars, rather than carbon being strongly enhanced (the latter is the case for CEMP- $s$ stars). Furthermore, the binarity properties of CEMP-no stars appear to be markedly different from the CEMP- $s$ class (Hansen, Andersen, \& Nordström 2013; Norris et al. 2013). Collectively, these observations suggest that most of the CEMP-no stars were probably born out of gas enriched by massive stars that ended their lives as Type II SNe with low levels of mixing and a high degree of fallback (Umeda \& Nomoto 2003; Ryan et al. 2005; Ishigaki et al. 2014).

However, there remains one aspect of this model that has yet to receive a satisfactory explanation - why does the fraction of CEMP-no stars become more common relative to nonCEMP stars at lower Fe abundance? Is it possible to observe a star that has $[\mathrm{Fe} / \mathrm{H}] \simeq-5.0$ and $[\mathrm{C} / \mathrm{Fe}] \simeq 0.0$ ? One possible solution to explain why no such star has been observed was proposed by Bromm \& Loeb (2003, see also Frebel et al. (2007)), who require a minimum level of $[\mathrm{C} / \mathrm{H}]$ and $[\mathrm{O} / \mathrm{H}]$ to be present in the birth cloud of the second generation

\footnotetext{
4 The 'CEMP' label is sometimes considered a misnomer, since some of the CEMP stars are believed to be underabundant in heavy elements, rather than enhanced in the low atomic number elements.
}

stars to allow efficient cooling by the fine-structure transitions of $\mathrm{C}_{\mathrm{II}}$ and $\mathrm{O}_{\mathrm{I}}$. In this picture, the cooling by $\mathrm{C}_{\mathrm{II}}$ and O I fine-structure lines allows the gas to fragment to smaller mass scales and form lower mass (hence longer lived) stars that have lived until the present day when we observe them. This model therefore implies that the increased fraction of CEMP-no stars at low metallicity is a selection effect, since we can only observe the lowest mass, second generation stars born out of gas with a minimum abundance of $\mathrm{C}$ and $\mathrm{O}$, irrespective of the $[\mathrm{Fe} / \mathrm{H}]$ abundance. This model was called into question with the discovery of the Leo star (Caffau et al. 2011; $[\mathrm{Fe} / \mathrm{H}]=-4.73,[\mathrm{C} / \mathrm{Fe}] \leq+0.93$ ), which is the most metal-deficient star currently known. To explain this almost primordial star, several authors have appealed to models of dust-induced fragmentation (Klessen, Glover, \& Clark 2012; Schneider et al. 2012; Ji, Frebel, \& Bromm 2014), which is able to assist low-mass star formation in the absence of high levels of C and O. In particular, Ji, Frebel, \& Bromm (2014) propose that both fine-structure cooling and fragmentation by dust are required in order to explain the population of CEMPno stars in addition to the Leo star.

In this paper, we present a simple solution to explain why carbon enhancements are more common at the lowest Fe abundance. Our interpretation is complementary to the methods described above, but does not rely on the detailed chemistry of the gas giving rise to the second generation of stars. We propose that the increased fraction of CEMP-no stars at lower metallicity is a selection effect due to an intimate link between the energy released by the SNe of the first stars and the host minihalo's ability to retain its gas: Qualitatively, a Type II SN with a high explosion energy is able to remove more $\mathrm{Fe}$ from the core of the massive star, and tends to produce a solar relative abundance of $\mathrm{C}$ to $\mathrm{Fe}($ i.e. $[\mathrm{C} / \mathrm{Fe}] \sim+0.0$ ). However, the high energy of such an explosion is more likely to evacuate the gas from the host minihalo thereby inhibiting the formation of second generation non-CEMP stars. On the other hand, low-energy SNe experience larger degrees of fallback which locks up the Fe in the compact remnant of the massive star, while still yielding a full complement of $\mathrm{C}$ (i.e. $[\mathrm{C} / \mathrm{Fe}] \gg+0.0)$. In this case, the low energy that is released by the $\mathrm{SN}$ is unable to evacuate the gas from the host potential, thereby allowing a generation of CEMP stars to form in the same minihalo, given sufficient time for the gas to cool.

In the following section, we provide the details of our chemical enrichment models for the minihalos that hosted the first stars. We present the main findings of our work in Section 3, where we explore the mass distribution of the halos that are able to retain their gas, the chemical properties of the second stars, and the fraction of CEMP stars that are born in the first galaxies. We present our main conclusions in Section 4. Throughout, we have assumed a Planck cosmology (Planck Collaboration et al. 2013), with $H_{0}=67.3 \mathrm{~km} \mathrm{~s}^{-1} \mathrm{Mpc}^{-1}, \Omega_{\mathrm{M}}=0.315$ and $\Omega_{\Lambda}=0.685$.

\section{MODEL SIMULATIONS}

The principal goal of this paper is to derive the metallicity and chemical abundance patterns for the second generation of stars in a cosmological context. In this section, we outline our model calculations that consider the full evolution of the first stars, and their impact on the gas confined by the host dark matter minihalos.

\subsection{Host Minihalo Properties}


Within the standard cold dark matter paradigm, detailed calculations of structure formation have shown that the first stars typically formed in minihalos of mass $\sim 10^{6} \mathrm{M}_{\odot}$ at redshifts $15 \lesssim z \lesssim 30$ (Tegmark et al. 1997; Abel, Bryan, \& Norman 2002; Bromm, Coppi, \& Larson 2002). This general picture has been confirmed by more recent work, where the minimum minihalo mass that is able to produce sufficient molecular hydrogen and therefore cool efficiently to successfully host a first generation of stars is $\sim 2 \times 10^{5} \mathrm{M}_{\odot}$ (e.g. Yoshida et al. 2003; O’Shea \& Norman 2007). We therefore start our calculations by drawing a random redshift and minihalo virial mass $\left(M_{200}\right)$ from the halo mass function derived by Reed et al. (2007) , with a lowermass cut-off at $\sim 2 \times 10^{5} \mathrm{M}_{\odot}$ in the redshift interval $15-30$. The halo concentration, $c_{200}$, is chosen by extrapolating the halo-mass-concentration relation derived by Prada et al. (2012), and is typically in the range $4-7$ for the halo masses and redshift range that we consider here. Prior to the formation of the first stars, we assume that the gas confined to the dark matter minihalo obeys an isothermal density profile, which is motivated by several cosmological simulations (e.g. Alvarez, Bromm, \& Shapiro 2006; O'Shea \& Norman 2007; Whalen et al. 2008). We assume the initial temperature of the gas is set by the virial temperature of the minihalo scaled (by a factor of 0.6 ) to match cosmological simulations (see e.g. Figure 19 of O'Shea \& Norman 2007), and is of the form:

$$
T_{\mathrm{gas}}(0)=0.6 \times 100^{1 / 3} \frac{\mu_{0} m_{\mathrm{H}}}{2 k_{\mathrm{B}}}\left(G M_{200} H\left(z_{\mathrm{coll}}\right)\right)^{2 / 3},
$$

where $H\left(z_{\text {coll }}\right)$ is the Hubble parameter at the minihalo collapse redshift $\left(z_{\text {coll }} \sqrt{6}\right.$, and $m_{\mathrm{H}}, k_{\mathrm{B}}$, and $G$ are the proton mass, Boltzmann constant and Newton gravitational constant respectively. The mean molecular weight in the initially neutral primordial gas is $\mu_{0} \simeq 1.23$.

\subsection{The First Stars}

In our study, we investigate zero-metallicity massive stars in the mass range $10-100 \mathrm{M}_{\odot}$ that would have ended their lives as Type-II core-collapse SN. In principle, the mass function of the first stars might extend to much higher masses, which we do not consider here for the following reasons: Stars more massive than $\sim 100 \mathrm{M}_{\odot}$ exhibit a pulsational instability due to the creation of electron/positron pairs in abundance (Ledoux 1941). Stars in the mass range $100-140 \mathrm{M}_{\odot}$ experience a series of such pulsations, causing the star to eject the outermost layers before it quietly collapses to a black hole (Woosley, Blinnikov, \& Heger 2007). Stellar evolution models of such stars have indicated that radiative mass loss provides negligible metal enrichment (Marigo, Chiosi, \& Kudritzki 2003). However, the pulsational winds from such stars might play a role in enriching early galaxies with $\mathrm{CNO}$, while producing no trace of the Fe-peak elements (Chen et al. 2014a; Woosley \& Heger 2014; but see Baraffe, Heger, \& Woosley 2001). In this context, detailed yield calculations for pulsational pair-instability $\mathrm{SNe}$ are highly desirable (see the discussion in Section 3.2). For stars in the mass range $\sim 140-260 \mathrm{M}_{\odot}$, the first instance of the pair-instability is sufficient to entirely disrupt the

5 The halo mass functions were generated using HMFcalc (Murray, Power, \& Robotham 2013).

${ }^{6} z_{\text {coll }}$ is defined to be the point at which the host minihalo's gas reaches high enough densities for the first stars to form - see O'Shea \& Norman (2007). star (Heger \& Woosley 2002; Chen et al. 2014b), leading to a pair-instability supernova (PISN) with a typical explosion energy of $\gtrsim 10^{52} \operatorname{erg} 7$; such a high explosion energy is sufficient to evacuate the host minihalo of all its gas, thereby prohibiting the formation of a second generation of stars in this minihalo (Greif et al. 2007; Whalen et al. 2008) - evacuating the gas reservoir defeats the primary goal of our study to investigate the halos that host the second generation of stars. Stars with mass $\gtrsim 260 \mathrm{M}_{\odot}$ are sufficiently massive that the onset of the pair-instability and the subsequent contraction and burning is unable to halt the gravitational collapse of the star, thus providing no metal enrichment (Fryer, Woosley, \& Heger 2001). In summary, metal-free stars in the mass range $10-100 \mathrm{M}_{\odot}$ are currently considered to be the dominant source of metal enrichment for the second stars, with perhaps an additional contribution from the pulsational pair-instability SNe.

We have thus adopted the model yield calculations performed by Heger \& Woosley (2010), which provide illustrative yields for 120 different stellar masses in the range $10-100 \mathrm{M}_{\odot}$ (with some models differing in mass by just $0.1 \mathrm{M}_{\odot}$ ). Each star was exploded with a piston located at the base of the oxygen shell (where the entropy per baryon $\sim 4 k_{\mathrm{B}}$ ), providing a final kinetic energy to the ejecta at infinity of either $0.3,0.6,0.9,1.2,1.5,1.8,2.4,3,5$, or 10 in units of $10^{51} \mathrm{erg}$. A simple prescription of the mixing between the stellar layers is implemented by a moving boxcar filter of a set width (14 different widths are considered here). Therefore, the final set of yields for the stars considered in our study totals 16,800 models. After drawing a stellar mass from the appropriate initial mass function (IMF; see below), we randomly draw an explosion energy and level of mixing from a uniform distribution. Given that we have no handle on the mixing and explosion properties of the first stars, our assumption allows sufficient flexibility to explore all of the available parameter space. In Figure 1, we present the IMF-weighted yields for illustration purposes, where we separately plot the integrated yields for SNe that release low energy $\left(0.3-0.6 \times 10^{51} \mathrm{erg}\right.$; blue curves), typical energies $\left(0.9-1.5 \times 10^{51} \mathrm{erg}\right.$; green curves), high energies $\left(1.8-3 \times 10^{51} \mathrm{erg}\right.$; orange curves $)$, and hypernovae $\left(5-10 \times 10^{51} \mathrm{erg}\right.$; red curves $)$.

The current generation of cosmological hydrodynamic simulations that follow the collapse and formation of the first stars suggest that either a single, binary, or small multiple of metal-free stars form in the center of dark matter minihalos (Turk, Abel, \& O'Shea 2009; Stacy, Greif, \& Bromm 2010; Stacy \& Bromm 2013; Hirano et al. 2014). We therefore assign each minihalo a single metal-free star (this assumption is relaxed in Section 2.6, where we allow small multiples of metal-free stars to form in a given dark matter minihalo), drawn from a flat stellar IMF, i.e. $\mathrm{d} N / \mathrm{d} m_{\star} \propto m_{\star}^{0}$, in the mass range $10-100 \mathrm{M}_{\odot}$. This assumption is supported by several recent numerical simulations (Stacy \& Bromm 2013; Hirano et al. 2014). However, the IMF of the first stars is still highly uncertain, and might instead represent a flat distribution in $\mathrm{d} N / \mathrm{d} \log m_{\star}$ (Greif et al. 2011). Thus, to demonstrate how our choice of a flat IMF (in $\mathrm{d} N / \mathrm{d} m_{\star}$ ) influences the results, we also consider the 'extreme' case of a Salpeter-like IMF for the first stars, in the mass range $10-100 \mathrm{M}_{\odot}$. For reference, the characteristic stellar masses for our chosen IMFs

\footnotetext{
7 We also note that there is a narrow range of stellar mass between 140 $150 \mathrm{M}_{\odot}$ where PISNe with explosion energies as low as $5 \times 10^{51}$ erg could exist.
} 

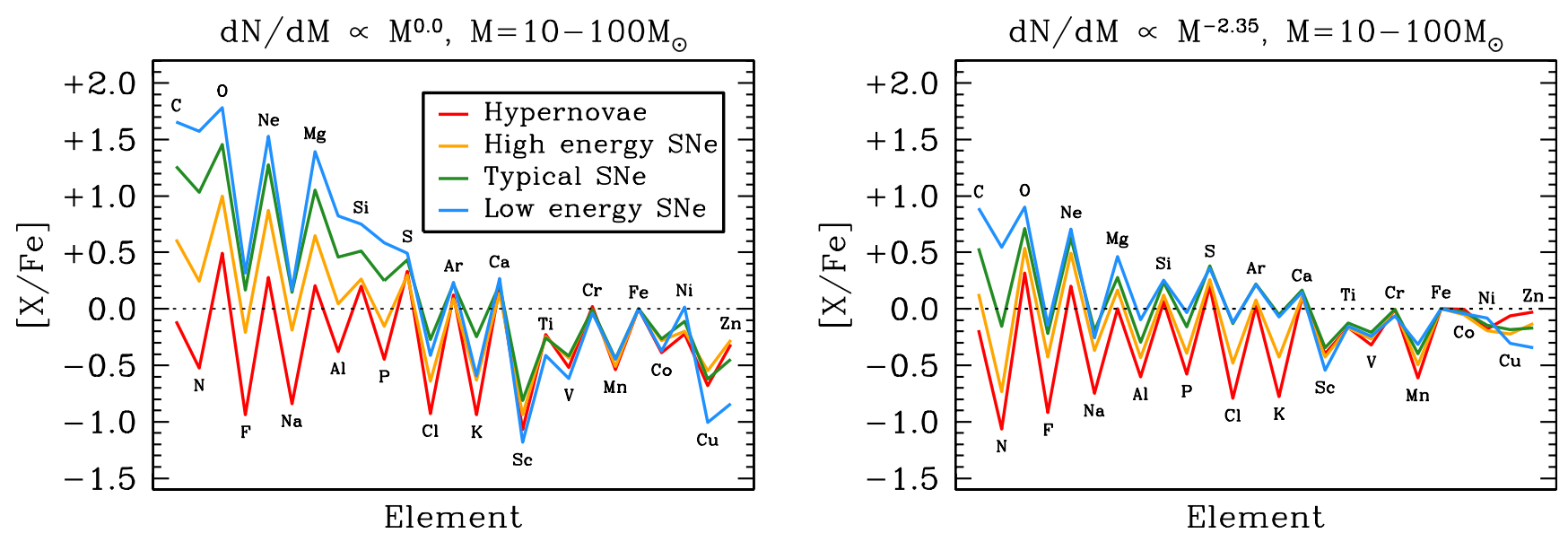

FIg. 1. - The IMF-weighted chemical yields for zero-metallicity stars with masses in the range $10-100 \mathrm{M}_{\odot}($ Heger \& Wooslev 2010$)$ are integrated over a flat IMF (left panel) and a Salpeter-like power-law IMF (right panel). For each IMF, we color-code the yields by the energy released during the explosion (see the text). This figure demonstrates that the highest mass massive stars coupled with low-energy explosions can produce a strong enhancement in the $\mathrm{C} / \mathrm{Fe}$ ratio, as noted previously (e.g. Umeda \& Nomoto 2003).

are $55 \mathrm{M}_{\odot}$ (flat IMF) and $22.3 \mathrm{M}_{\odot}$ (Salpeter-like IMF).

We also note that the initial mass function of the first stars could be very different from the mass function of stars that provide metal enrichment; there may be several windows in stellar mass where massive stars collapse directly to a black hole and eject no metals (Woosley \& Heger 2014). If such a scenario is realized, the mass function of the first stars that eject metals may resemble a broken picket fence, rather than a power law. For this study, we assume that every star born in the mass range $10-100 \mathrm{M}_{\odot}$ provides metal enrichment.

\subsection{Presupernova $\mathrm{H}$ II Region Properties}

The first stars can have a profound impact on their environment during their lives. They emit copious quantities of $\mathrm{H}$-ionizing photons which serve to ionize and thereby heat the surrounding gas to $\sim 10^{4} \mathrm{~K}$. After the first stars turn on, the initial (isothermal) density distribution characterized by the temperature $T_{\text {gas }}(0)$ becomes overpressured and causes the gas to expand. The expansion is bounded by an isothermal shock, known as a "champagne" flow, and is characterised by the self-similar solutions presented by Shu et al. (2002). The subsequent time-evolution of the radial gas density distribution for a self-gravitating isothermal cloud of gas is given by

$$
\rho(r, t)=\frac{k_{\mathrm{m}} \alpha(x)}{4 \pi G t^{2}} \quad x=\frac{r}{c_{\mathrm{s}}(0) t}
$$

where $c_{s}(0)=k_{\mathrm{B}} T_{\text {gas }}(0) / \mu_{0} m_{\mathrm{H}}$ is the sound speed of the gas before the first stars are born. The constant $k_{\mathrm{m}}=$ $M_{\text {gas }} / 0.6 M_{200} \simeq 0.17$ is chosen so that the total mass of baryons inside the virial radius matches that observed in cosmological hydrodynamic simulations (see e.g. Table 2 from O'Shea \& Norman 2007). After the first stars are born, they heat the center of the cloud to a new temperature, $T_{\text {gas }}(t)$, with a corresponding sound speed $c_{s}(t)=k_{\mathrm{B}} T_{\text {gas }}(t) / \mu_{\text {ion }} m_{\mathrm{H}}$, where $\mu_{\text {ion }}=0.59$ is the mean molecular weight for fully ionized primordial gas. By numerically integrating the Shu et al. (2002) equations, the reduced central density, $\alpha(x=0)$, in Equation2 can be directly related to the ratio of the initial and final sound speeds of the gas, $\epsilon=c_{s}(0)^{2} / c_{s}(t)^{2}$. To minimize computational time, we generate a fine grid of $\alpha(0)$ values, numerically integrate the Shu et al. (2002) equations and compute the corresponding values for $\epsilon$. Then, given the initial and final sound speeds of the gas in each minihalo, we interpolate the $\alpha(x)$ values.

The boost in thermal energy that is given to the gas by a star during its life can be estimated by equating the heating rate to the cooling rate of a pure $\mathrm{H}$ gas (i.e. assuming thermal equilibrium in a smooth medium). The cooling rate that we use includes a contribution from recombination cooling as well as collisional excitation and ionization cooling (see Cen 1992 for the relevant fitting formulae that we have adopted herein). Assuming that recombinations balance photoionizations, the photoelectron heating rate is then simply

$$
\frac{\Gamma_{\mathrm{pe}}}{n_{\mathrm{H}} n_{e}}=\alpha_{\mathrm{B}} \psi k_{\mathrm{B}} T_{\text {eff }}
$$

where $\alpha_{\mathrm{B}}$ is the case B recombination coefficient for $\mathrm{H}, \psi$ is the mean photoelectron energy, and $T_{\text {eff }}$ is the effective temperature of the star. To calculate $T_{\text {eff }}$ for our full range of stellar masses, we have generated the following fitting formula for a metal-free star with mass $m_{\star}$ based on the detailed computations by Schaerer (2002):

$$
T_{\text {eff }}=6600 \mathrm{~K} \times\left(m_{\star} / \mathrm{M}_{\odot}\right)^{1.09-0.257 \log _{10}\left(m_{\star} / \mathrm{M}_{\odot}\right)}
$$

The mean photoelectron energy can then be calculated by assuming that the first stars radiated a blackbody spectrum, $B_{v}\left(T_{\text {eff }}\right)$, with color temperature $T_{\text {eff }}$

$$
\psi k_{\mathrm{B}} T_{\text {eff }}=\frac{\int_{v_{0}}^{\infty}\left[B_{v}\left(T_{\text {eff }}\right) / h v\right] \sigma_{v}(\mathrm{H})\left(h v-h v_{0}\right) \mathrm{d} v}{\int_{v_{0}}^{\infty}\left[B_{v}\left(T_{\text {eff }}\right) / h v\right] \sigma_{v}(\mathrm{H}) \mathrm{d} v}
$$

where $\sigma_{\nu}(\mathrm{H})$ is the photoionization cross-section for $\mathrm{H}$, and $h v_{0}=13.6 \mathrm{eV}$ is the photoionization energy of $\mathrm{H}$.

The gas density profile at the end of the star's life is defined by Equation 2] where the time variable corresponds to the main sequence lifetime of a Population III star. To estimate the main-sequence lifetime for the range of stellar masses we consider here, we have generated the following fitting formula, which is derived from the computations by Schaerer (2002):

$$
t_{\mathrm{ms}}=1300 \mathrm{Myr} \times\left(m_{\star} / \mathrm{M}_{\odot}\right)^{-2.407+0.5323 \log _{10}\left(m_{\star} / \mathrm{M}_{\odot}\right)}
$$

An example density profile for a 15 and $40 \mathrm{M}_{\odot}$ star occupying a $7 \times 10^{5} \mathrm{M}_{\odot}$ minihalo that collapsed at redshift $z=$ 24 is shown in Figure 2] The choice of these parameters 


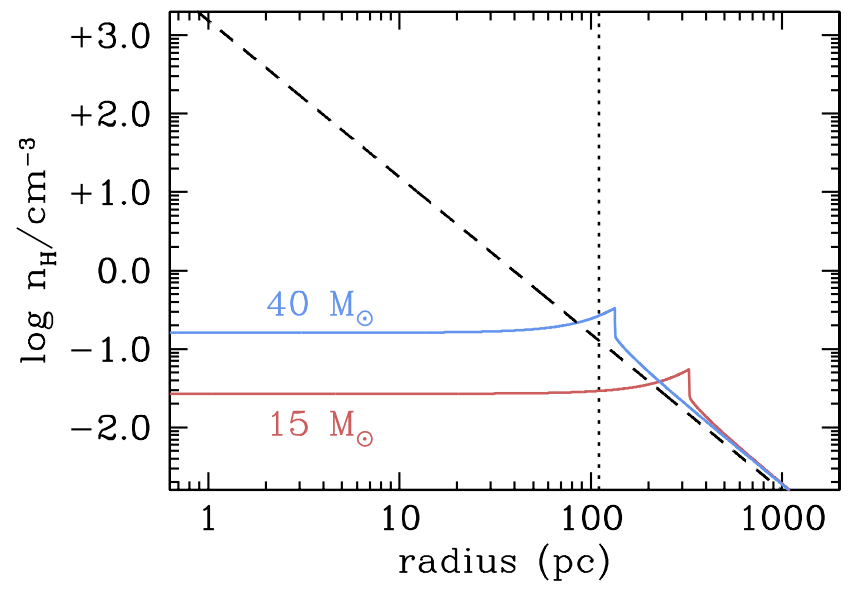

Fig. 2.- The gas density profile for a $M_{200}=7 \times 10^{5} \mathrm{M}_{\odot}$ minihalo that collapsed at redshift $z=24$ is shown as the dashed line. The blue and red lines show the gas density profile of this minihalo after being heated for the duration of a $40 \mathrm{M}_{\odot}$ and $15 \mathrm{M}_{\odot}$ metal-free star's life, respectively. Note the striking similarity between our model calculations and that presented in Figures 1 and 2 of Whalen et al. (2008, see also Abel, Wise, \& Bryan 2007; Yoshida et al. 2007). The virial radius of this minihalo is marked by the vertical dotted line.

allow for a direct comparison with Figures 1 and 2 from Whalen et al. (2008). Although we have not modeled the presupernova evolution using computationally expensive full three-dimensional (3D) cosmological hydrodynamic simulations, the striking agreement between our calculations and other, more expensive simulations (e.g. Abel, Wise, \& Bryan 2007; Yoshida et al. 2007; Whalen et al. 2008) demonstrates that we have captured the relevant physics of the presupernova evolution for the first stars, and the feedback they imparted on the surrounding gas. Thus, the computational simplicity of our models allows us to explore vast regions of parameter space in a fraction of the time.

\subsection{Blastwave Evolution}

The final stage of our modeling procedure accounts for the Type II core-collapse SN blastwave at the end of a star's life. Greif et al. (2007) have shown that the blastwave evolution for the first stars is well-approximated by an energy and momentum conserving formalism. When including the host potential, however, this calculation becomes computationally expensive Ostriker \& McKee 1988 8 . Given that the binding energy of the host minihalo is comparable to the energy released for some of our adopted models (see Section 2.2), we have instead calculated the stall radius, $r_{\text {stall, }}$, which corresponds to the radius when the shock velocity is comparable to local sound speed of the gas. Specifically,

$$
\frac{E_{\text {sh }}\left(r_{\text {stall }}\right)}{M_{\text {sw }}\left(r_{\text {stall }}\right)}=\frac{k_{\mathrm{B}} T_{\text {gas }}\left(r_{\text {stall }}\right)}{\mu\left(r_{\text {stall }}\right) m_{\mathrm{H}}}
$$

where the kinetic energy of the shock in the thin shell approximation is given by

$$
E_{\mathrm{sh}}\left(r_{\mathrm{stall}}\right)=\left(1-f_{\mathrm{r}}\right) E_{\mathrm{exp}}-\Delta W / 2 .
$$

\footnotetext{
8 We note that in the work of Greif et al. (2007), the host potential could be safely ignored, since the kinetic energy released during the explosion was much larger than the binding energy of the halo.
}

Here

$$
\Delta W=\int_{0}^{r_{\mathrm{stall}}} G\left[M_{\mathrm{dm}}(<r)+M_{\mathrm{sw}}(r)\right]\left(\frac{M_{\mathrm{sw}}(r)}{r^{2}}-4 \pi \rho\left(r, t_{\mathrm{ms}}\right)\right) \mathrm{d} r
$$

is the change in the total gravitational potential produced by the blastwave, and $f_{\mathrm{r}}=0.7$ is the fraction of the kinetic energy released by the core-collapse $\mathrm{SN}$ explosion $\left(E_{\text {exp }}\right)$ that is radiated away in the Sedov-Taylor phase (Ostriker \& McKee 1988). The dark matter mass interior to a radius $r$ (assumed to be static) and the swept up gas mass are respectively given by:

$$
\begin{aligned}
M_{\mathrm{dm}}(<r) & =M_{200} \frac{\ln (1+u)-u /(1+u)}{\ln \left(1+c_{200}\right)-c_{200} /\left(1+c_{200}\right)} \\
M_{\mathrm{sw}}(r) & =4 \pi \int_{0}^{r} \rho\left(R, t_{\mathrm{ms}}\right) R^{2} \mathrm{~d} R
\end{aligned}
$$

where $u=r c_{200} / r_{200}$ and $r_{200}$ is the virial radius.

\subsection{Gas Retention and Metallicity}

The metallicity of the enriched gas depends on how efficiently the metals are mixed with the pristine material swept up by the blastwave. For relatively high-mass minihalos $(\sim$ $\left.10^{8} \mathrm{M}_{\odot}\right)$, the current state-of-the-art simulations that include a prescription for metal diffusion indicate that the gas is efficiently mixed within $\sim 300 \mathrm{Myr}$ (Greif et al. 2010). For minihalos of lower mass, efficient mixing takes place within several tens of Myr (Bland-Hawthorn, Sutherland, \& Karlsson 2011; Ritter et al. 2012). For now, we assume that the ejected metals are efficiently mixed with the swept up pristine material interior to the stall radius, such that the gas will be enriched to a metal abundance:

$$
[\mathrm{Z} / \mathrm{H}]=\log _{10}\left(\frac{m_{\mathrm{H}}}{m_{\mathrm{Z}}} \frac{M_{\mathrm{Z}}}{X M_{\mathrm{sw}}\left(r_{\text {stall }}\right)}\right)-\log _{10}(\mathrm{Z} / \mathrm{H})_{\odot}
$$

where $m_{\mathrm{H}}$ and $m_{\mathrm{Z}}$ are the atomic masses of hydrogen and element ' $Z$ ' respectively, $M_{Z}$ refers to the total ejected mass of element $Z$ from the metal-free star and $X=0.75$ is the primordial mass fraction of $\mathrm{H}$. The solar values for all elements, marked by the $\odot$ symbol, are taken from Asplund et al. (2009). We further consider the possibility of inefficient mixing in Section 3.2

In order to form a second generation of stars encoded with the chemical signature of the first stellar generation, we require that the blastwave does not evacuate the gas from the host dark matter minihalo. Specifically, we assume that all minihalos where the blastwave lifts the gas to beyond the virial radius will not form a second generation of stars before merging into more massive parent systems. Thus, the only halos that will form a second generation of stars are those where $r_{\text {stall }}<r_{200}$. Although the retention or evacuation of gas from a minihalo can only be correctly treated within a 3D setting (Ritter et al. 2012; Smith et al. 2014; Ritter et al. 2014), our prescription allows us to explore large areas of parameter space that would otherwise be impossible with full 3D hydrodynamic simulations. In any case, we can test the impact of this assumption, by considering a stricter limit where the only minihalos that retain their gas are those where the blastwave does not lift the gas beyond half the virial radius (see Section 3.2.

\subsection{Population III Multiples}


Recent simulations suggest that a given minihalo may form a binary or small multiple of massive metal-free stars (Turk, Abel, \& O’Shea 2009; Stacy, Greif, \& Bromm 2010; Greif et al. 2012; Stacy \& Bromm 2013; Hirano et al. 2014), which could reduce the chance of gas retention relative to the single star scenario (K.-J. Chen et al. in preparation). To simulate the chemical enrichment from a small multiple of Population III stars in a single dark matter minihalo, we draw one, two, three, or four stars from the stellar IMF under consideration, and assume that all stars form at a redshift $z_{\text {coll }}$ (i.e. the star formation occurs in a "burst"). The temperature of the $\mathrm{H}$ II region heated by this small multiple is calculated as described above, but replacing $B_{v}\left(T_{\text {eff }}\right)$ in Equation 5 by the sum over every star in the minihalo, $\Sigma_{i} B_{v}\left(T_{\mathrm{eff}, \mathrm{i}}\right)$. The blastwave is initiated after the maximum stellar lifetime has passed (i.e. the least massive star that exploded). For multiple stars, the blastwave is followed as an 'equivalent' single explosion as described in Section 2.4 specifically, the total energy of the blastwave is equal to the sum of the energy released from the stellar multiple. This assumption is the limiting case where it is most difficult for a minihalo to retain its gas. Similarly, each individual element yield is totaled for all stars in a minihalo, so that we track the total yield of, for example, carbon from the stellar population.

Thus, our calculation ignores the physics of SN kicks that might occur after the most massive star in a binary explodes (Conroy \& Kratter 2012). We note that if Population III runaways are efficiently kicked from the center of the dark matter potential, they will produce offcentered explosions, which provide an enhanced gas retention (Bland-Hawthorn, Sutherland, \& Karlsson 2011).

\subsection{Model Statistics}

For the results of this paper, we are concerned with the halos that are able to retain their gas after forming the first generation of stars. To provide reliable statistics, we continuously simulate minihalos for a given IMF and multiple of stars until we have uncovered at least 10,000 models that retain their gas.

\section{RESULTS}

In this section, we present the results from our chemical enrichment models of the first stars. As expected, the results of our simulations depend strongly on the energy released by the SNe. To demonstrate the effect of the explosion energy, we have divided the models into four bins depending on the average energy of the SN explosion in a minihalo; the intervals that we have selected are based on the grid of kinetic energy used in the nucleosynthesis models (Heger \& Woosley 2010, see Section 2.2). Specifically, the blue curves in Figures 36 show low-energy SNe $\left(0.3-0.75 \times 10^{51} \mathrm{erg}\right)$, the green curves show typical SNe $\left(0.75-1.65 \times 10^{51} \mathrm{erg}\right)$, the orange curves are for high-energy SNe $\left(1.65-4 \times 10^{51} \mathrm{erg}\right)$ and the red curves represent the hypernovae $\left(4-10 \times 10^{51} \mathrm{erg}\right)$. For a given $\mathrm{SN}$ energy, we have assumed that all values of the stellar mixing are equally probable.

\subsection{Halo Mass Distribution of the First Galaxies}

The working definition of a "first galaxy", in its most basic form, is a dark matter halo that confines a long-lived stellar population (see e.g. the review by Bromm \& Yoshida 2011). Within our model, the first galaxies would be those that are the least disrupted by the SN explosion from the first stars, corresponding to the halos that are able to retain their gas.
There are two primary mechanisms in our models that influence the distribution of retained halo masses. Obviously, the most important factor is the energy that is available to lift the gas out of the minihalo, which largely determines the shape of the retained halo mass distribution. However, the form of the primordial stellar IMF also influences the probability that a minihalo will retain its gas, since the presupernova gas distribution strongly depends on stellar masses. For the example shown in Figure 2, a $15 \mathrm{M}_{\odot}$ star, despite having a lower luminosity than the $40 \mathrm{M}_{\odot}$ star, drastically reduces the central gas density of the minihalo during its relatively longer life. At a fixed minihalo mass, it is easier for a $\mathrm{SN}$ to evacuate the gas from the minihalos that host the longest-lived massive stars. Therefore, a minihalo of a given mass is more likely to retain its gas if the form of the primordial stellar IMF is top-heavy.

In Figure 3, we present the distribution of halo masses that retain their gas (color-coded by the average energy of the hosted $\mathrm{SNe}$ ). For reference, we also show the halo mass function from which these models are drawn as a thick black curve. It is immediately obvious that there is a significant deficit of low-mass halos that are able to retain their gas. Given that the least disrupted minihalos are those that are able to promptly form a second generation of stars, our calculations suggest that the second stars were formed in halos of mass $\sim$ a few $\times 10^{6} \mathrm{M}_{\odot}$.

\subsection{Chemistry}

The chemistry that is born into the second stars is highly sensitive to both the stellar IMF and the energy released during the SN explosion of a metal-free star - these are the two primary mechanisms that also drive the distribution of retained halo masses. This implies that there may be a strong selection bias when interpreting the nature of the first stars by studying their chemical yields in the stellar atmospheres of the most metal-poor second generation stars in our Galaxy. The SNe that evacuated the gas from their host minihalo would have been unable to encode their metal yields in a second generation of stars. For example, there are no environments currently known where the distinct chemical signatures of PISNe (Heger \& Woosley 2002) have been unambiguously detected. This could be a consequence of an observational survey bias, where the primary goal is to identify the most metal-poor second generation stars; PISNe are expected to enrich the surrounding medium to a metallicity of 1/100 solar (Salvadori, Schneider, \& Ferrara 2007; Karlsson, Johnson, \& Bromm 2008), which is a considerably higher metallicity than that targeted by current surveys for metal-poor stars. In addition to this bias, we suggest that the high energy of their SN explosion would have evacuated the gas from their host minihalo, resulting in their chemical signature being rarely incorporated into a subsequent stellar population (see Greif et al. 2007; Whalen et al. 2008). More commonly, one should expect to find the chemical signatures from the $\mathrm{SNe}$ of massive stars that experienced core-collapse, which typically release more than an order of magnitude less energy than PISNe. Indeed, the chemical signatures of corecollapse $\mathrm{SNe}$ are regularly observed in the lowest metallicity stars in the halo of our Galaxy (e.g. McWilliam et al. 1995; Cayrel et al. 2004; Lai et al. 2008; Heger \& Woosley 2010; Roederer et al. 2014).

The strong link between the metal yield, explosion energy, and gas retention suggests that the second stars should exhibit a strong, systematic variation in their relative element abundances. Naively, one should expect that lower atomic number 


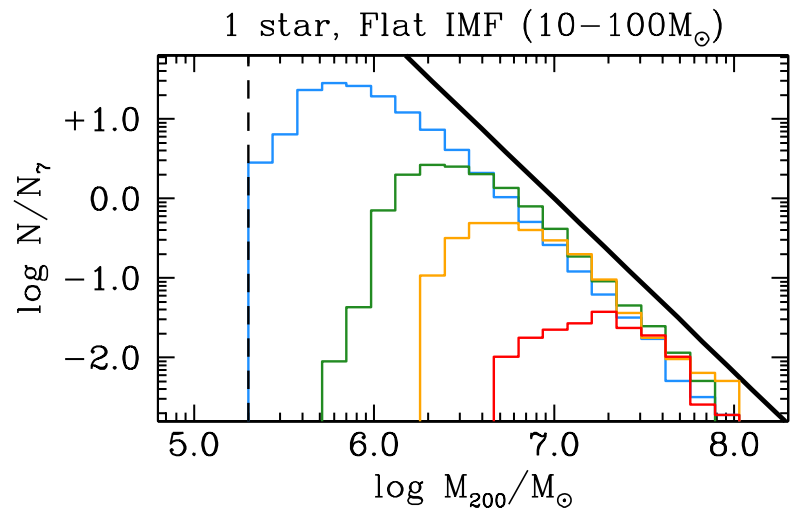

1 star, Salpeter IMF $\left(10-100 \mathrm{M}_{\odot}\right)$
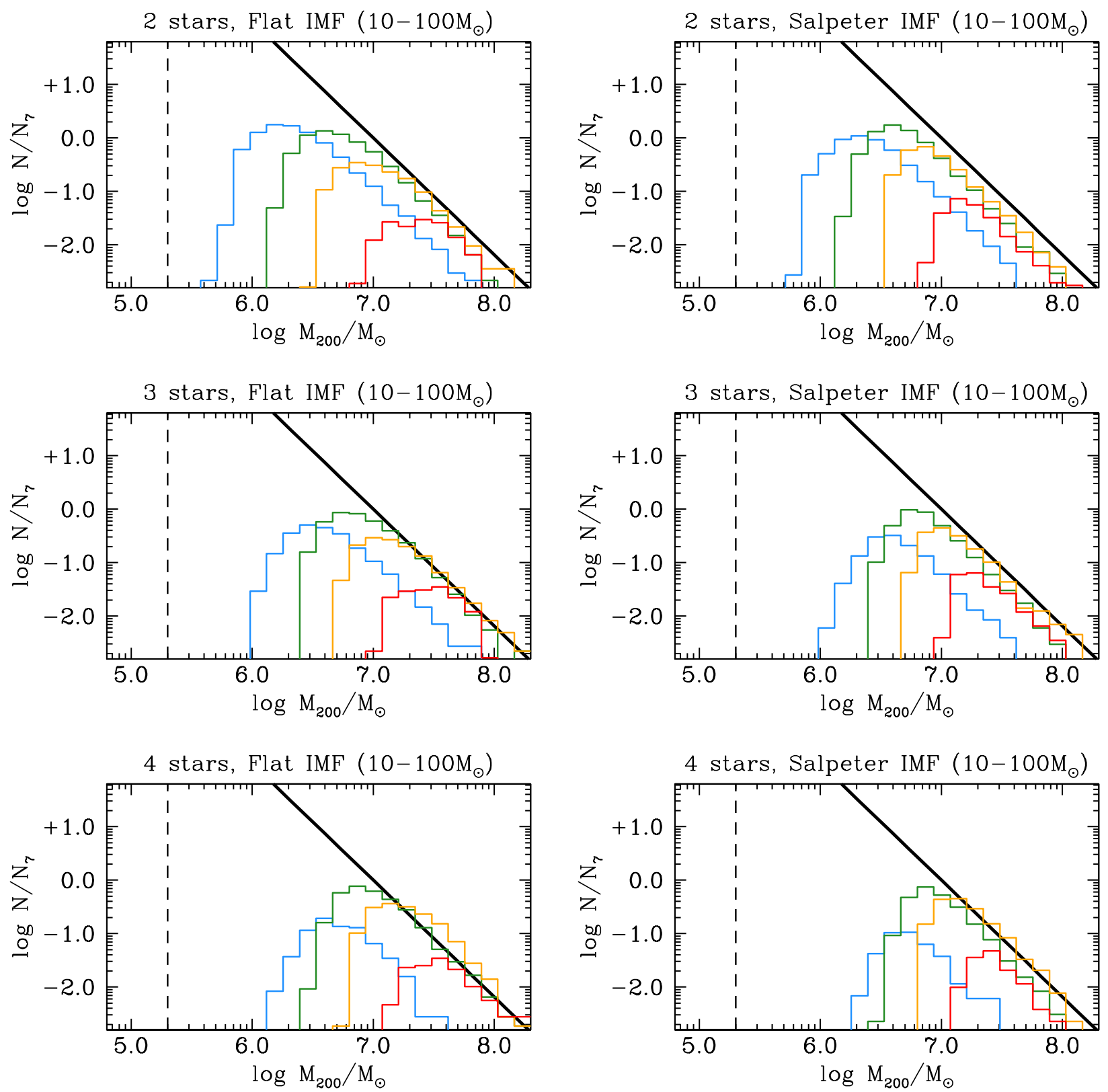

FIG. 3.- Distribution of halo masses that retain their gas after hosting a first generation of stars are shown, color-coded by the average energy released by the $\mathrm{SNe}$ in a given minihalo; blue, green, and orange represent the low-, typical, and high-energy SNe, while the red curves are for hypernovae. For reference, the thick black curve illustrates the sampled halo mass function. The distributions are normalized to the total number of retained models at $10^{7} \mathrm{M}_{\odot}$. Each row shows the four cases considered here, where a given minihalo hosts one, two, three, or four massive metal-free stars. The left panels illustrate the retained halo mass function for stars with a flat IMF, while the right panels are for a Salpeter-like IMF (both IMFs cover the mass range $10-100 \mathrm{M}_{\odot}$ ). Minihalos below $2 \times 10^{5} \mathrm{M}_{\odot}$ (indicated by the vertical dashed line) are unable to cool sufficiently to form a first stellar generation (Yoshida et al. 2003; O’Shea \& Norman 2007). 

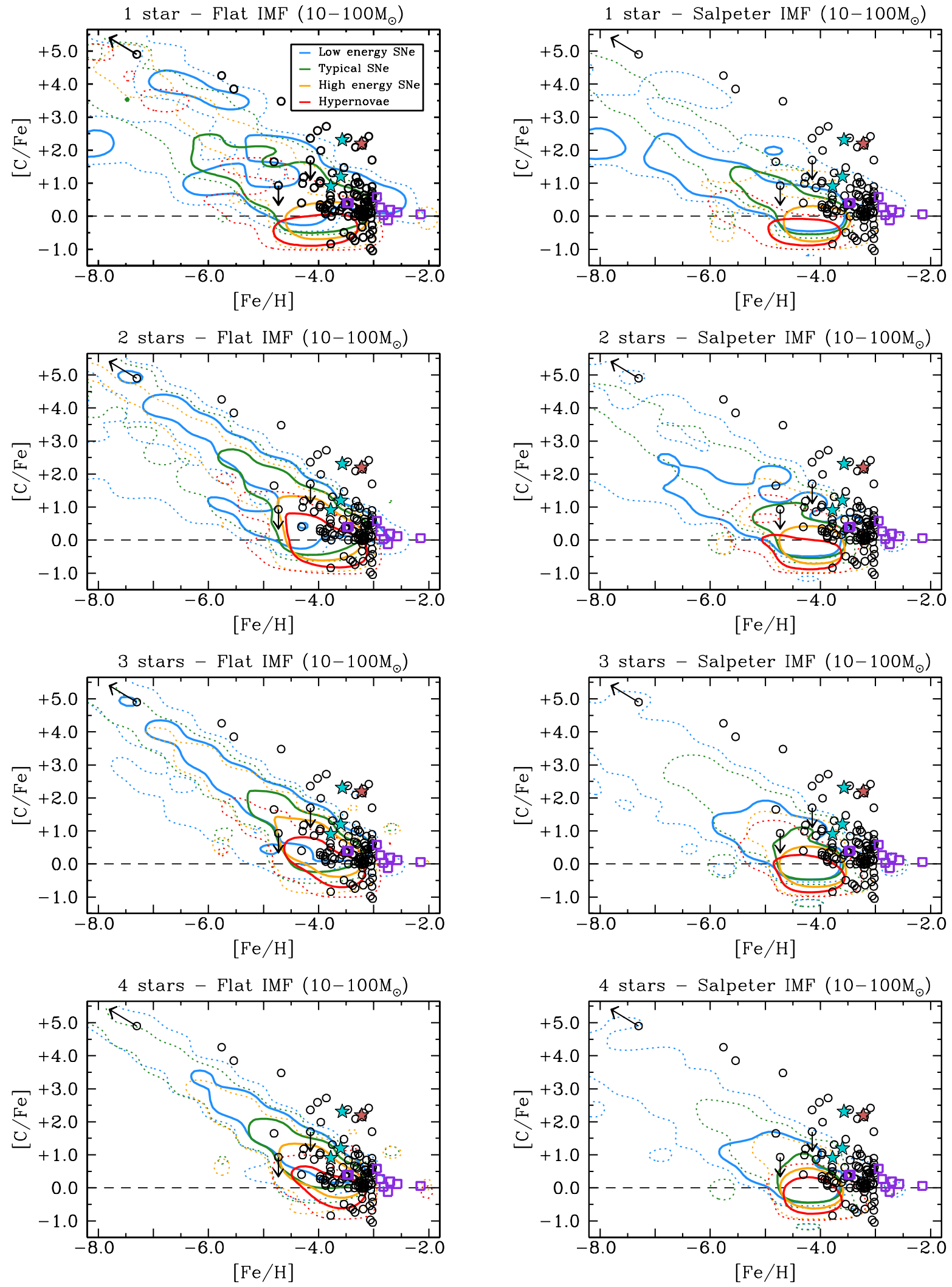

FIG. 4.- $[\mathrm{C} / \mathrm{Fe}]$ vs. $[\mathrm{Fe} / \mathrm{H}]$ abundances for the halos that retain their gas after hosting a first generation of stars. The left and right panels display the distribution of values for a flat and Salpeter-like IMF respectively. From top to bottom, each panel shows the four cases considered here, where each minihalo hosts one, two, three, or four massive metal-free stars. Each set of models is separated based on the average energy of all SNe for a given minihalo, corresponding to SNe with: low energy (blue; $0.3-0.75 \times 10^{51} \mathrm{erg}$ ), typical energy (green; $0.75-1.65 \times 10^{51} \mathrm{erg}$ ), high energy (orange; $1.65-4 \times 10^{51} \mathrm{erg}$ ) and hypernovae (red; $4-10 \times 10^{51} \mathrm{erg}$ ). The solid (dotted) curves enclose $68(95)$ per cent of the models for each explosion energy. The black circles are the measurements from Milky Way stellar halo stars modeled in one dimension and assuming local thermodynamic equilibrium (Yong et al. 2013; Aoki et al. 2013; Roederer et al. 2014; Hansen et al. 2014). The two very low-metal stars reported by Caffau et al. (2011) and Keller et al. (2014), and one star from Hansen et al. (2014), are shown with their derived limits. We also plot four CEMP-no stars that are associated with the Segue 1 (blue stars; Norris et al. 2010, Frebel, Simon, \& Kirby |2014) and Boötes I (red star; Lai et al. 2011, Gilmore et al. 2013) dwarf spheroidal galaxies. The purple squares are for the most metal-poor damped Lyman- $\alpha$ systems Cooke et al.2011b, 2014a b). The solar level of [C/Fe] is marked by the black horizontal dashed line. 

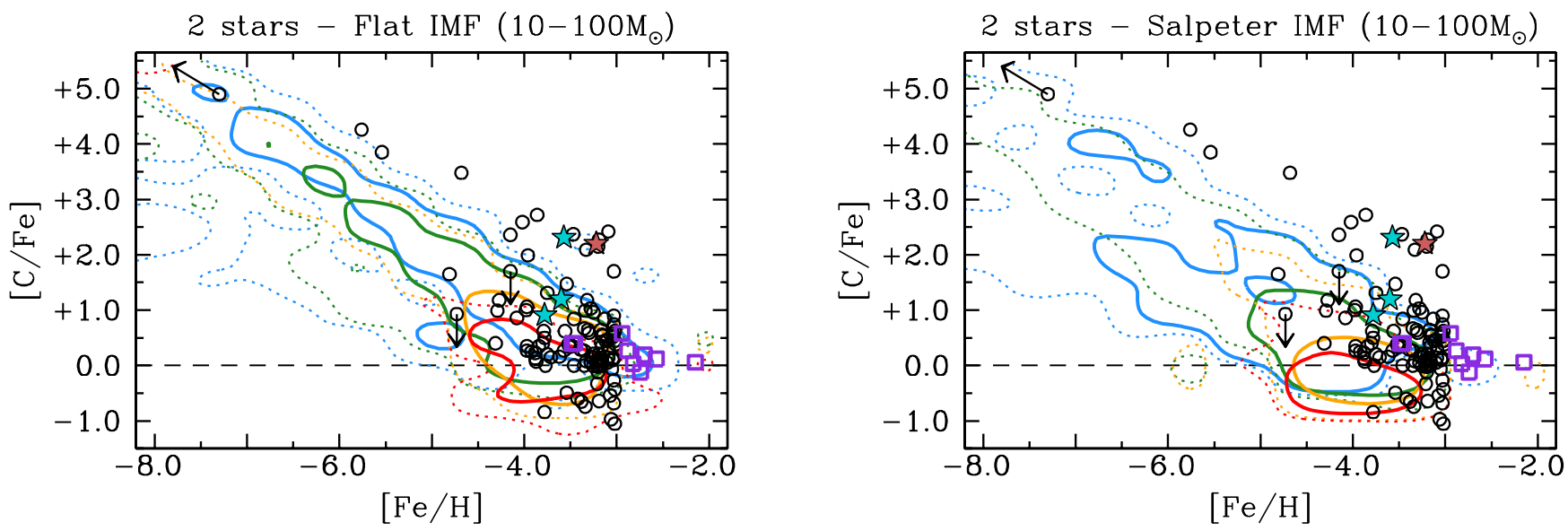

FIg. 5. - Same as Figure 4 however, in this plot we only consider the minihalos where the SN blastwave does not lift the gas to beyond half the virial radius. We only illustrate the case where two stars (i.e. a Population III binary) forms per minihalo; the remaining cases for one, three, or four stars per minihalo are qualitatively similar to their respective panels in Figure 4

nuclei (e.g. C) are overabundant relative to the higher atomic number nuclei (e.g. Fe), since the latter are synthesized in the innermost, tightly bound core of a massive star. In Figure 4 we show the distribution of $[\mathrm{C} / \mathrm{Fe}]$ and $[\mathrm{Fe} / \mathrm{H}]$ values for the halos that are able to retain their gas, color-coded by the average energy released by the $\mathrm{SNe}$ in the host minihalo. The solid and dotted contours enclose 68 and 95 per cent of the models, respectively. We also overplot the stellar data compiled by Yong et al. (2013) and Roederer et al. (2014). We have ignored stars from these samples with $[\mathrm{Ba} / \mathrm{Fe}]>0.0$, which are probably enriched with the $s$-process. We supplement these data with three CEMP-no stars from Aoki et al. (2013), four stars from Hansen et al. (2014), and the two metal-poor halo stars reported by Caffau et al. (2011) and Keller et al. (2014). We have also included a small handful of stars from the Milky Way ultra-faint dwarf spheroidal galaxies, including three stars from Segue 1 (Norris et al. 2010; Frebel, Simon, \& Kirby 2014) and one star from Boötes I (Lai et al. 2011; Gilmore et al. 2013).

In general, the variation in $[\mathrm{C} / \mathrm{Fe}]$ as a function of the $\mathrm{Fe}$ abundance for our models show a good agreement with the trend exhibited by the most metal-poor stars, especially given that there are no free parameters in our models. In particular, our calculations match the currently available data for the most Fe deficient CEMP stars, while simultaneously matching the Leo star (Caffau et al. 2011) and most of the "carbonnormal' stars just below $[\mathrm{Fe} / \mathrm{H}] \simeq-3.0$. All minihalos are enriched to a total metallicity $[\mathrm{Z} / \mathrm{H}] \simeq-3.0$, which is in good agreement with cosmological hydrodynamic simulations (e.g. Wise et al. 2012). We further note that the most Fe-deficient CEMP stars need not have formed from the products of a single SN; our models demonstrate that the most Fe-deficient CEMP stars can also be produced by a small multiple of first stars.

In Figure 4, there is also a small cluster of $\sim 12$ 'super' CEMP stars that are offset from our models toward higher $[\mathrm{C} / \mathrm{Fe}]$ and higher $[\mathrm{Fe} / \mathrm{H}]$. This small handful of stars is clearly distinct from both our model calculations and from observations of other CEMP stars at a similar metallicity. In addition, these stars are found in both the Milky Way halo and satellite dwarf galaxies. One possibility is that these stars may have formed in minihalos that were enriched by early episodes of AGB nucleosynthesis (Salvadori \& Ferrara 2012). A similar possibility is that these low-metallicity CEMP-no stars may have hosted an AGB binary companion, and therefore represent the 'low- $s$ ' extremely metal-poor counterparts of the CEMP-s population (Masseron et al. 2010). This scenario is supported by models of AGB nucleosynthesis at $[\mathrm{Fe} / \mathrm{H}]=$ -3.0 , which predict a $[\mathrm{C} / \mathrm{Fe}]$ abundance in the range +2.0 to +3.0 (Campbell \& Lattanzio 2008). These models are consistent with the level of enrichment observed for the super CEMP stars. Dedicated radial velocity monitoring for this sample of stars is now required to confirm if they are members of binary systems. At present, radial velocity variations for 5 of these 12 stars have already been measured (Starkenburg et al. 2014), 3 of which show evidence of binarity?

Alternatively, this handful of CEMP stars could have been born out of gas that was enriched by metal-free stars exploding as Type-II SNe, as considered herein, with a boost in the CNO elements provided by stars that yield no Fe-peak elements. Several models do entertain stellar yields with no Fe-peak elements, including intermediate mass Population III stars (Chieffi et al. 2001; Herwig 2005; Campbell \& Lattanzio 2008), models with rotationally enhanced mass loss from massive stars (Meynet, Ekström, \& Maeder 2006; Hirschi 2007; Meynet et al. 2010), and massive stars that end their lives as a pulsational pair instability $\mathrm{SNe}$ (with progenitor masses in the range $\sim 100-140 \mathrm{M}_{\odot}$, Woosley \& Heger 2014).

On the other hand, if this cluster of stars does not represent a distinct population, their disagreement with our models might be a consequence of our assumption that the metal yields from the first stars are uniformly mixed with the swept up pristine material. Inefficient mixing has recently been proposed as a possible explanation for the elevated, constant $[\alpha / \mathrm{Fe}]$ ratios seen for several stars over a broad range of metallicity in the Segue 1 ultra-faint dwarf spheroidal galaxy (Frebel, Simon, \& Kirby 2014). If mixing is considered to be less efficient than allowed by our fiducial model, the contours in Figure 4 would be inflated to account for regions of relatively higher and lower metallicity. However, inefficient mixing is unable to explain the absence of stars between the 'ordinary' CEMP stars and the 'super' CEMP stars. The details of the mixing process can only be addressed with more realistic

9 The stars that exhibit binarity are HE 1150-0428, CS 22957-027, and J1422+0031. The two stars with no present evidence for binarity are J1613+5309 and Segue 1-7. The remaining seven stars have undetermined radial velocity variations: HE 0233-0343, HE 1012-1540, HE 1310-0536, HE 2139-5432, CS 22958-042, CS 29498-043, and Boo21 (Starkenburg et al. 2014; Hansen et al. 2014). 
3D, cosmological hydrodynamic simulations.

We also note that the $[\mathrm{Fe} / \mathrm{H}]$ abundance that we derive from our models depends on the amount of pristine $\mathrm{H}$ gas swept up by the blastwave, which is fixed by the energy delivered by the $\mathrm{SN}$ and the potential of the host minihalo. Our fiducial model (see Section 2.5) requires that the gas must not be lifted to beyond the virial radius in order for the minihalo to retain its gas. If we relax this criterion to allow models where the blastwave stalls at twice the virial radius, the distributions of the models presented in Figure 4 would be shifted toward lower $[\mathrm{Fe} / \mathrm{H}]$, since 'additional' pristine gas will be swept up. Similarly, if we were to enforce a stricter retention criterion, the models shift toward higher $[\mathrm{Fe} / \mathrm{H}]$. We explore this possibility in Figure 5 for the case where two stars (i.e. a Population III binary) are born per minihalo, and require that each minihalo is only able to retain its gas if the SN blastwave does not extend beyond half the virial radius. Enforcing a stricter criterion results in only a minor shift to the overall distribution of $[\mathrm{C} / \mathrm{Fe}]$ and $[\mathrm{Fe} / \mathrm{H}]$ values. Our retention criterion is therefore relatively insensitive to the conclusions drawn here regarding the origin of CEMP stars at low metallicity. As a final note, our models have also assumed that stellar multiplicity is independent of the minihalo mass. In principle, the multiplicity of the first stars may depend on the thermal evolution of the collapsing cloud, which in turn depends on the mass of the minihalo (e.g. Hirano et al. 2014). However, this should not significantly affect the trend of $[\mathrm{C} / \mathrm{Fe}]$ with $[\mathrm{Fe} / \mathrm{H}]$; for a given IMF, our simulation results exhibit a general similarity in the $[\mathrm{C} / \mathrm{Fe}]-[\mathrm{Fe} / \mathrm{H}]$ plane, independent of the stellar multiplicity.

\subsection{The Fraction of Carbon-Enhanced Metal-Poor stars}

The number of stars that exhibit an enhancement in carbon relative to those stars that do not is known to be much higher at lower Fe abundance (Beers \& Christlieb 2005; Carollo et al. 2012; Yong et al. 2013; Carollo et al. 2014). In the extremely metal-poor regime $([\mathrm{Fe} / \mathrm{H}] \lesssim-3.0)$, the dominant class of these carbon-enhanced stars are those that exhibit normal abundances of their neutron-capture elements (Aoki et al. 2007; Carollo et al. 2014). In this section, we use our suite of realistic chemical enrichment models from the first stars to estimate the fraction of CEMP stars at the lowest metallicities. In doing so, we assume that all values of the 'mixing' parameter from the Heger \& Woosley (2010) metal-free nucleosynthesis models are equally probable. We further assume that each minihalo that is able to retain its gas will form a second generation of stars. Using the definition proposed by Aoki et al. (2007), we define our models to be carbonenhanced when $[\mathrm{C} / \mathrm{Fe}] \geq+0.7$. The fraction of our models that produce carbon-enhanced minihalos as a function of the Fe abundance is displayed in Figure 6 for the two IMFs under consideration. Using the same criterion, we have calculated the observed fraction of carbon-enhanced halo stars from the data sample described in Section 3.2. excluding the four stars that have been observed in the Segue 1 and Boötes I dwarf spheroidal galaxies.

A general prediction of our models - independent of the IMF and gas mixing efficiency - is that all future stars that are discovered with an $\mathrm{Fe}$ abundance $[\mathrm{Fe} / \mathrm{H}] \lesssim-5.0$ should almost certainly exhibit a carbon enhancement. Before drawing conclusions from the CEMP fraction at somewhat higher metallicities, we caution that some of the stars in the observational sample may not have been exclusively enriched by Population III stars; some of the CEMP stars with $[\mathrm{Fe} / \mathrm{H}] \lesssim-3.0$ may have seen pollution from an extinct AGB companion star, or otherwise may have condensed from gas that was also enriched by low-metallicity Population II stars. These two possibilities would respectively under- or overestimate the observed CEMP fraction relative to our models. Despite these potential caveats, our models succeed in producing a decrease in the CEMP fraction toward higher $[\mathrm{Fe} / \mathrm{H}]$ that is in broad agreement with the current observational data.

\section{SUMMARY AND CONCLUSIONS}

We have developed a simple formalism with no free parameters to investigate the detailed chemical enrichment signatures from the first stars. We propose that there is a strong link between the chemistry born into the second stars, and the host minihalo's ability to retain its gas reservoir following the $\mathrm{SNe}$ from the first stars. Qualitatively, massive metal-free stars that produce high-energy $\mathrm{SNe}$ are able to eject significant $\mathrm{Fe}$ from their core, but will also efficiently evacuate the gas from their host minihalo thereby inhibiting the formation of the second stars. On the other hand, minihalos that host low-energy SNe are able to retain their gas reservoir but, as a consequence, yield very little Fe from the highly bound core of the Pop III star. In both cases, high-energy and low-energy $\mathrm{SNe}$ eject a full complement of the lower atomic number elements, such as $\mathrm{C}$, which are less bound to the massive star. The net result is to produce a significant number of Fe-poor second generation stars that are highly rich in the low atomic number elements, in accord with the present observations of the most Fe-poor stars in the halo of the Milky Way. On the basis of our detailed suite of calculations that explore an extensive region of parameter space for the properties of the first stars and their host minihalos, we draw the following main conclusions.

(1) In addition to the energy released by the first stars, the primordial stellar IMF plays an important role in determining whether or not a minihalo will retain its reservoir of gas, and the resulting chemistry of the second generation of stars. The longest-lived massive metal-free stars dramatically reduce the central gas density distribution during their (relatively) long life. As a consequence, the gas is displaced to larger radii which indirectly assists the supernova blastwave in evacuating the gas from the host minihalo.

(2) The dark matter halos that are the least disrupted by the first stars will also be the first environments to host a second stellar generation. In this context, CEMP stars would have been among the first Population II stars to have formed in the first galaxies. Our calculations also suggest that the chemistry that seeded the CEMP stars was in place at very early times and in relatively low-mass halos (a few $\times 10^{6} \mathrm{M}_{\odot}$ ).

(3) We propose that some carbon-enhanced stars that contain normal abundances of their neutron-capture elements (the socalled CEMP-no stars) are the by-product of an intimate link between the explosions of the first stars and their host minihalo's ability to retain its gas. Our suite of models are able to reproduce the general dependence of $[\mathrm{C} / \mathrm{Fe}]$ with $[\mathrm{Fe} / \mathrm{H}]$, as well as the variation in the fraction of CEMP stars relative to non-CEMP stars as a function of the Fe abundance.

(4) It is commonly believed that the most Fe-deficient Galactic halo stars were enriched by just a single metal-free star. Although our calculations are certainly consistent with this picture, one cannot rule out the possibility that a small multiple of stars contributed to the enrichment of these second generation Fe-poor stars.

(5) The present lack of chemical evidence for very massive stars $\left(\gtrsim 140 \mathrm{M}_{\odot}\right)$ does not suggest they were uncommon or 

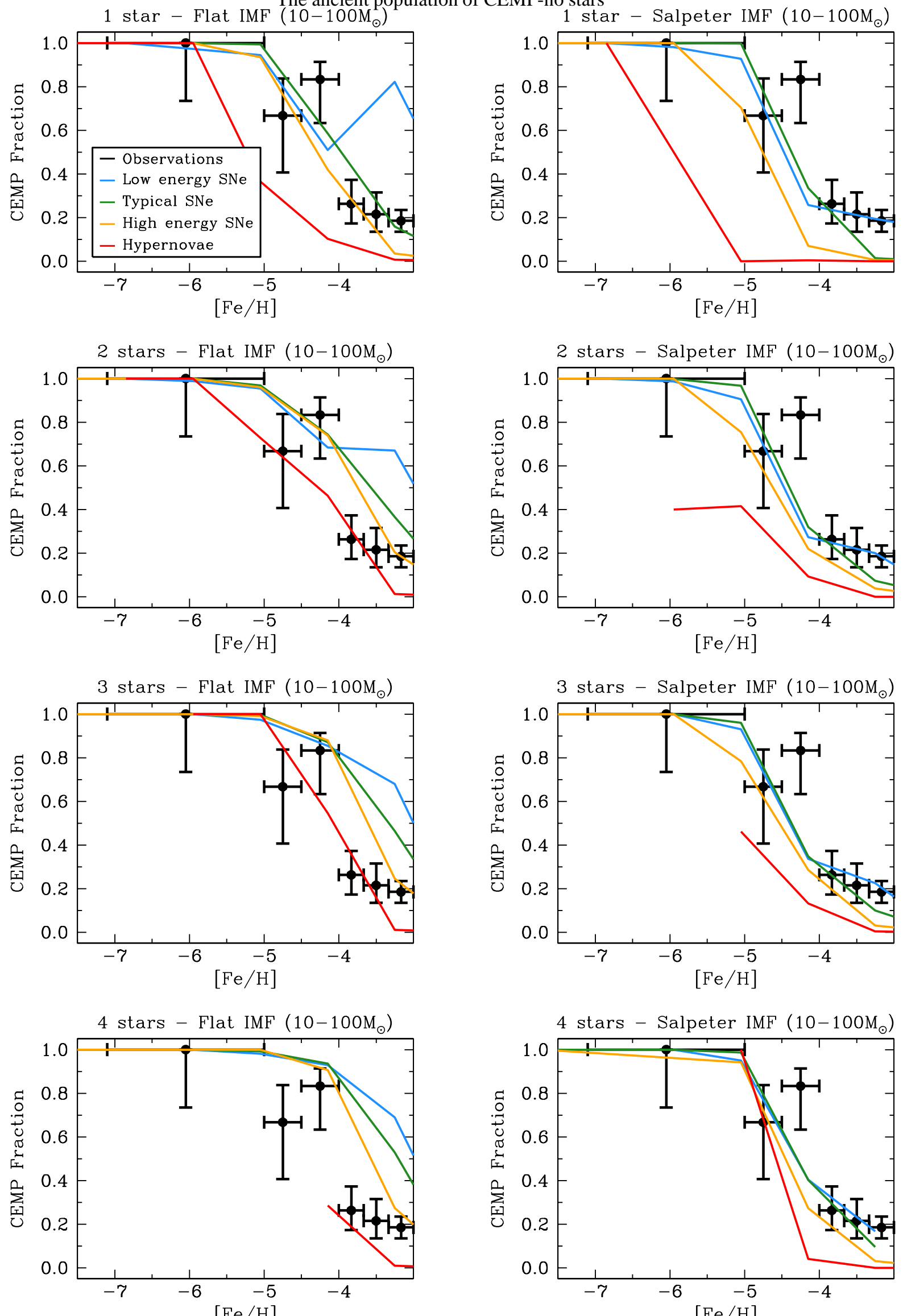

FIG. 6.- Fraction of CEMP stars at a given Fe metallicity is shown for the halos that retain their gas after hosting a first generation of stars (same layout and color coding as Figure 4). The black points are primarily based on the compilations by Yong et al. (2013) and Roederer et al. (2014), in addition to three stars from (Aoki et al. 2013), three stars from Hansen et al. (2014), and the two stars reported by Caffau et al. (2011) and Keller et al. (2014). We assume the Aoki et al. (2007) definition of CEMP stars (i.e. $[\mathrm{C} / \mathrm{Fe}] \geq+0.7$ for our models). For a given explosion energy, the model curves are truncated at the model with the lowest $[\mathrm{Fe} / \mathrm{H}]$. 
did not exist. Rather, the large energy released during their SN explosion could have inhibited their products from being incorporated into subsequent stellar populations.

Our models suggest that the first stars commonly enrich some halos to $1 / 1000$ of solar metallicity, similar to some of the most metal-poor damped Lyman- $\alpha$ systems already known at redshift $z \sim 3$ (Pettini et al. 2008; Cooke et al. $2011 \mathrm{a}$ ). Therefore, understanding the physical properties of these near-pristine neutral gas reservoirs, may provide key clues for understanding the star formation environments of the second stars (Cooke et al. 2014b). Finally, the recent discovery of carbon-enhanced Fe-poor stars in both the Segue 1 and Boötes I Milky Way dwarf spheroidal galax- ies (Norris et al. 2010; Lai et al. 2011; Gilmore et al. 2013; Frebel, Simon, \& Kirby 2014), adds further support to the idea that these systems are the surviving relics of the first galaxies (Bovill \& Ricotti 2009; Salvadori \& Ferrara 2009; Muñoz et al. 2009).

\section{ACKNOWLEDGMENTS}

We thank Anna Frebel, Miho Ishigaki, Max Pettini, Stefania Salvadori, Michele Trenti, and Stan Woosley for their advice and helpful discussions on various aspects of this paper. We are grateful to an anonymous referee who made valuable suggestions that improved this paper. R. J. C. is partially supported by NSF grant AST-1109447. P. M. acknowledges support by the NSF through grant OIA-1124453 and by NASA through grant NNX12AF87G.

\section{REFERENCES}

Abel T., Bryan G. L., Norman M. L., 2002, Sci, 295, 93

Abel T., Wise J. H., Bryan G. L., 2007, ApJ, 659, L87

Alvarez M. A., Bromm V., Shapiro P. R., 2006, ApJ, 639, 621

Aoki W., Beers T. C., Christlieb N., Norris J. E., Ryan S. G., Tsangarides S., 2007, ApJ, 655, 492

Aoki W., et al., 2013, AJ, 145, 13

Asplund M., Grevesse N., Sauval A. J., Scott P., 2009, ARA\&A, 47, 481

Baraffe I., Heger A., Woosley S. E., 2001, ApJ, 550, 890

Beers T. C., Christlieb N., 2005, ARA\&A, 43, 531

Bland-Hawthorn, J., Sutherland, R., Karlsson, T. 2011, in A Universe of

Dwarf Galaxies, ed. M. Koleva, P. Prugniel, I. Vauglin (EAS Publications

Series, Vol. 48; Les Ulis: EDP Sciences), 397

Bovill M. S., Ricotti M., 2009, ApJ, 693, 1859

Bromm V., Coppi P. S., Larson R. B., 2002, ApJ, 564, 23

Bromm V., Loeb A., 2003, Nature, 425, 812

Bromm V., Yoshida N., Hernquist L., 2003, ApJ, 596, L135

Bromm V., Yoshida N., 2011, ARA\&A, 49, 373

Bromm V., 2013, Rep. Prog. Phys., 76, 112901

Caffau E., et al., 2011, Nature, 477, 67

Campbell S. W., Lattanzio J. C., 2008, A\&A, 490, 769

Carollo D., et al., 2012, ApJ, 744, 195

Carollo D., Freeman K., Beers T., Placco V., Tumlinson J., Martell S., 2014, arXiv, arXiv:1401.0574

Cayrel R., et al., 2004, A\&A, 416, 1117

Cen R., 1992, ApJS, 78, 341

Chen K.-J., Woosley S., Heger A., Almgren A., Whalen D., 2014a, arXiv, arXiv:1402.4134

Chen K.-J., Heger A., Woosley S., Almgren A., Whalen D., 2014b, arXiv, arXiv: 1402.5960

Chieffi A., Domínguez I., Limongi M., Straniero O., 2001, ApJ, 554, 1159

Christlieb N., et al., 2002, Nature, 419, 904

Cohen J. G., et al., 2006, AJ, 132, 137

Conroy C., Kratter K. M., 2012, ApJ, 755, 123

Cooke R., Pettini M., Steidel C. C., Rudie G. C., Jorgenson R. A., 2011a, MNRAS, 412, 1047

Cooke R., Pettini M., Steidel C. C., Rudie G. C., Nissen P. E., 2011b, MNRAS, 417, 1534

Cooke R., Pettini M., Murphy M. T., 2012, MNRAS, 425, 347

Cooke R. J., Pettini M., Jorgenson R. A., Murphy M. T., Steidel C. C., 2014a, ApJ, 781, 31

Cooke R. J., Pettini M., Jorgenson R. A., 2014b, arXiv:1406.7003

Ellison S. L., Prochaska J. X., Hennawi J., Lopez S., Usher C., Wolfe A. M., Russell D. M., Benn C. R., 2010, MNRAS, 406, 1435

Frebel A., et al., 2005, Nature, 434, 871

Frebel A., Christlieb N., Norris J. E., Thom C., Beers T. C., Rhee J., 2007, ApJ, 660, L117

Frebel A., Simon J. D., Kirby E. N., 2014, ApJ, 786, 74

Fumagalli M., O’Meara J. M., Prochaska J. X., 2011, Sci, 334, 1245

Fryer C. L., Woosley S. E., Heger A., 2001, ApJ, 550, 372

Gilmore G., Norris J. E., Monaco L., Yong D., Wyse R. F. G., Geisler D., 2013, ApJ, 763, 61

Greif T. H., Johnson J. L., Bromm V., Klessen R. S., 2007, ApJ, 670, 1

Greif T. H., Glover S. C. O., Bromm V., Klessen R. S., 2010, ApJ, 716, 510

Greif T. H., Springel V., White S. D. M., Glover S. C. O., Clark P. C., Smith R. J., Klessen R. S., Bromm V., 2011, ApJ, 737, 75
Greif T. H., Bromm V., Clark P. C., Glover S. C. O., Smith R. J., Klessen

R. S., Yoshida N., Springel V., 2012, MNRAS, 424, 399

Haiman Z., Thoul A. A., Loeb A., 1996, ApJ, 464, 523

Hansen T., Andersen J., Nordström B., 2013, Proceedings of the XII

International Symposium on Nuclei in the Cosmos (NIC XII),

Proceedings of Science, 146, 193

Hansen T., et al., 2014, ApJ, 787, 162

Heger A., Woosley S. E., 2002, ApJ, 567, 532

Heger A., Woosley S. E., 2010, ApJ, 724, 341

Herwig F., 2005, ARA\&A, 43, 435

Hirano S., Hosokawa T., Yoshida N., Umeda H., Omukai K., Chiaki G.,

Yorke H. W., 2014, ApJ, 781, 60

Hirschi R., 2007, A\&A, 461, 571

Ishigaki M. N., Tominaga N., Kobayashi C., Nomoto K., 2014, arXiv, arXiv: 1404.4817

Ji A. P., Frebel A., Bromm V., 2014, ApJ, 782, 95

Karakas A. I., Lattanzio J. C., 2014, arXiv, arXiv:1405.0062

Karlsson T., Johnson J. L., Bromm V., 2008, ApJ, 679, 6

Keller S. C., et al., 2014, arXiv, arXiv:1402.1517

Klessen R. S., Glover S. C. O., Clark P. C., 2012, MNRAS, 421, 3217

Komiya Y., Habe A., Suda T., Fujimoto M. Y., 2010, ApJ, 717, 542

Lai D. K., Bolte M., Johnson J. A., Lucatello S., Heger A., Woosley S. E., 2008, ApJ, 681, 1524

Lai D. K., Lee Y. S., Bolte M., Lucatello S., Beers T. C., Johnson J. A., Sivarani T., Rockosi C. M., 2011, ApJ, 738, 51

Ledoux P., 1941, ApJ, 94, 537

Limongi M., Chieffi A., 2012, ApJS, 199, 38

Lucatello S., Tsangarides S., Beers T. C., Carretta E., Gratton R. G., Ryan S. G., 2005, ApJ, 625, 825

Lucatello S., Beers T. C., Christlieb N., Barklem P. S., Rossi S., Marsteller B., Sivarani T., Lee Y. S., 2006, ApJ, 652, L37

Madau P., Ferrara A., Rees M. J., 2001, ApJ, 555, 92

Maio U., Khochfar S., Johnson J. L., Ciardi B., 2011, MNRAS, 414, 1145

Marigo P., Chiosi C., Kudritzki R.-P., 2003, A\&A, 399, 617

Masseron T., Johnson J. A., Plez B., van Eck S., Primas F., Goriely S., Jorissen A., 2010, A\&A, 509, A93

McClure R. D., 1985, J. Roy. Astron. Soc. Can., 79, 277

McWilliam A., Preston G. W., Sneden C., Searle L., 1995, AJ, 109, 2757

Meynet G., Ekström S., Maeder A., 2006, A\&A, 447, 623

Meynet G., Hirschi R., Ekstrom S., Maeder A., Georgy C., Eggenberger P., Chiappini C., 2010, A\&A, 521, A30

Muñoz J. A., Madau P., Loeb A., Diemand J., 2009, MNRAS, 400, 1593

Murray S., Power C., Robotham A., 2013, arXiv, arXiv:1306.6721

Norris J. E., Christlieb N., Korn A. J., Eriksson K., Bessell M. S., Beers

T. C., Wisotzki L., Reimers D., 2007, ApJ, 670, 774

Norris J. E., Gilmore G., Wyse R. F. G., Yong D., Frebel A., 2010, ApJ, 722, L104

Norris J. E., et al., 2013, ApJ, 762, 28

O’Shea B. W., Norman M. L., 2007, ApJ, 654, 66

Ostriker J. P., McKee C. F., 1988, RvMP, 60, 1

Pettini M., Zych B. J., Steidel C. C., Chaffee F. H., 2008, MNRAS, 385, 2011

Planck Collaboration, et al., 2013, arXiv, arXiv:1303.5076

Prada F., Klypin A. A., Cuesta A. J., Betancort-Rijo J. E., Primack J., 2012,

MNRAS, 423, 3018 
Reed D. S., Bower R., Frenk C. S., Jenkins A., Theuns T., 2007, MNRAS, 374, 2

Ritter J. S., Safranek-Shrader C., Gnat O., Milosavljević M., Bromm V., 2012, ApJ, 761, 56

Ritter, J. S., Sluder, A., Safranek-Shrader, C., Milosavljevic, M., and Bromm, V., 2014, in preparation

Roederer I. U., Preston G. W., Thompson I. B., Shectman S. A., Sneden C., Burley G. S., Kelson D. D., 2014, arXiv, arXiv:1403.6853

Ryan S. G., Aoki W., Norris J. E., Beers T. C., 2005, ApJ, 635, 349

Salvadori S., Ferrara A., 2009, MNRAS, 395, L6

Salvadori S., Ferrara A., 2012, MNRAS, 421, L29

Salvadori S., Schneider R., Ferrara A., 2007, MNRAS, 381, 647

Scannapieco E., Schneider R., Ferrara A., 2003, ApJ, 589, 35

Schaerer D., 2002, A\&A, 382, 28

Schneider R., Omukai K., Limongi M., Ferrara A., Salvaterra R., Chieffi A., Bianchi S., 2012, MNRAS, 423, L60

Shu F. H., Lizano S., Galli D., Cantó J., Laughlin G., 2002, ApJ, 580, 969

Simcoe R. A., Sullivan P. W., Cooksey K. L., Kao M. M., Matejek M. S., Burgasser A. J., 2012, Nature, 492, 79

Smith B. D., Wise J. H., O'Shea B. W., and Norman M. L., in preparation
Stacy A., Bromm V., 2013, MNRAS, 433, 1094

Stacy A., Greif T. H., Bromm V., 2010, MNRAS, 403, 45

Starkenburg E., Shetrone M. D., McConnachie A. W., Venn K. A., 2014, arXiv, arXiv:1404.0385

Tegmark M., Silk J., Rees M. J., Blanchard A., Abel T., Palla F., 1997, ApJ, 474, 1

Tominaga N., Umeda H., Nomoto K., 2007, ApJ, 660, 516

Tumlinson J., 2006, ApJ, 641, 1

Turk M. J., Abel T., O’Shea B., 2009, Sci, 325, 601

Umeda H., Nomoto K., 2003, Nature, 422, 87

Whalen D., van Veelen B., O’Shea B. W., Norman M. L., 2008, ApJ, 682, 49

Wise J. H., Abel T., 2008, ApJ, 685, 40

Wise J. H., Turk M. J., Norman M. L., Abel T., 2012, ApJ, 745, 50

Woosley S. E., Blinnikov S., Heger A., 2007, Nature, 450, 390

Woosley S. E., Heger A., 2014, in Very Massive Stars in the Local Universe, ed. J. S. Vink (Springer), in press

Yong D., et al., 2013, ApJ, 762, 27

Yoshida N., Abel T., Hernquist L., Sugiyama N., 2003, ApJ, 592, 645

Yoshida N., Oh S. P., Kitayama T., Hernquist L., 2007, ApJ, 663, 687 